\title{
The influence of neuromuscular activation on the estimation of optimal muscle length using a muscle model
}

\author{
Yang Hua Lin ${ }^{\mathrm{a}, *}$ and Tung-Wu Lu ${ }^{\mathrm{b}}$ \\ ${ }^{a}$ Department of Physical Therapy, Chang Gung University, Kweishan, Taoyuan, Taiwan \\ ${ }^{\mathrm{b}}$ Graduate Institute of Biomedical Engineering, National Taiwan University, Taipei, Taiwan
}

\begin{abstract}
Objective. This investigation aims to determine whether optimal muscle length was estimated with joint toque generated by maximal voluntary isometric muscle contraction (MVIC), which differed from that was estimated with joint torques generated through electrical stimulation superimposed on MVIC (MVIC $\mathrm{ES}_{\text {). }}$.

Design. An experimental investigation was conducted to measure joint torques of the elbow joint in the conditions with MVIC and with MVICES. The measured joint torques were used to examine the level of neuromuscular activation under MVIC condition as well as to estimate the optimal muscle length of elbow flexors by using in a theoretical muscle model with various conditions of muscle contraction.

Background. Usually muscle model parameters such as maximum muscle force, optimal length are estimated by the joint torques measured during MVIC, assuming maximum level of neuromuscular activation. However, several experimental studies have shown that MVIC are in fact submaximal contraction and cannot represent as complete neuromuscular activation. As a result, the use of MVIC for the estimation of muscle model parameters may not be appropriately able to produce satisfactory results. Methods. Eight subjects first performed MVIC of their elbow flexors. Electrical stimulation was superimposed to MVIC to induce true maximal contraction of the muscles. The resulting joint torques were measured in each test condition and used to calculated the optimal muscle lengths by a muscle model combined with an optimization procedure. Comparisons of the results for the two conditions were made with paired $\mathrm{T}$ test.

Results. The joint torques produced by electrically stimulated the elbow flexors at the elbow joint were statistically higher than those produced by maximal voluntary contraction. Optimal muscle lengths estimated through the joint torques generated by MVIC with electrical stimulation were statistically different from those estimated without electrical stimulation. The optimal lengths of the elbow flexors were calculated with adjusting the neuromuscular activation to be submaximal level and showed no statistically difference from that estimated with superimposed electric stimulation.

Conclusion. Joint torques used for estimating optimal muscle lengths are better derived from electrical stimulation superimposed on MVIC of the relevant muscles or MVIC accompanied with adjusting the level of neuromuscular activation in the range of 0.62 to 0.93 .
\end{abstract}

Keywords: Maximal voluntary isometric contraction, superimposed electric stimulation, neuromuscular activation, optimal muscle length

\footnotetext{
* Address for correspondence: Yang Hua Lin, Ph.D, Department of Physical Therapy, Chang Gung University, 259, Wen-Hwa 1st Rd., Kweishan, Taoyuan, Taiwan, 333. Tel.: +886 33283016 5438; Fax: +886 3 3283031; E-mail: linyh@mail.cgu.edu.tw.
}

\section{Introduction}

Muscles exert force to produce movements. The force generation of muscles determines the movement control and loading effects on skeletal system. However, in vivo determination of the forces transmitted by the muscles is difficult due to technological and ethical 
consideration. Therefore, mathematical models combined with non-invasive experimental measurements have been used to estimate such forces. The ability of these models in successfully predicting muscle forces depends not only on the models themselves but also model parameters including length, velocity and maximal neuromuscular activation. In 1894 Blix first [1] described the relationship between the lengths of frog muscles and the maximal forces exerted. The maximal force of a muscle also depends on the instantaneous rate of change in length. Force-length relations as well as force-velocity relations enable the muscles to meet the functional demands imposed during everyday activities.

Force-length and force-velocity relations are the attributes of active tension of a muscle. In a mathematical model of length-tension relationship reported by Kaufman et al. [2], one of the parameters representing the mechanical and architectural properties is the optimal length of an active muscle $\left(\mathrm{L}_{0}\right)$. The optimal length of a muscle is directly related to the estimation of the force-velocity properties of intact human skeletal muscles [3]. Hence, optimal length is an important feature to determine the active tension of a muscle.

Optimal lengths of various skeletal muscles have been determined via experimental measurements in vitro as well as mathematical calculations in vivo. For example, photographic techniques have been proposed for in vitro measurements [4]. The optimal lengths of wrist muscles have been measured intraoperatively by laser diffraction [5] or derived in vivo by examining the length-tension relationship through electrical stimulation [6,7]. In a recent study, Chang et al. [8] proposed an optimization approach for the estimation of optimal muscle length. A model of the length-tension relationship of the following form was used

$$
F=\left(\alpha \cdot F_{l}+F_{p e}\right) \cdot P C S A \cdot \sigma
$$

where $\alpha$ represents the level of neuromuscular activation; $F_{l}$ is the normalized active muscle tension; $F_{p e}$ is the normalized passive muscle tension; $P C S A$ is physiologic cross-section area; $\sigma$ is muscle.

Experimental determination of the force-length relationship of a muscle requires maximal muscle activation under isometric conditions. Usually, maximal voluntary isometric contraction (MVIC) is used to represent maximal muscle activation, setting to unity [3]. In consequence, $\alpha$ was set as one assuming maximal muscular activation. Maximal activation in involving intact human skeletal muscles is often assumed as maximal voluntary isometric efforts (MVIC). However, previ- ous studies have shown that electrical stimulation superimposed on MVIC (MVIC ${ }_{\mathrm{ES}}$ ) elicits greater muscle torques than MVIC [9-11] so MVIC represents a submaximal muscle contraction. The prediction of optimal muscle lengths using torques produced by MVIC and assuming the level of neuromuscular activation as unity is subjected to doubt.

This investigation aimed to study (1) the range of level of neuromuscular activation during MVIC, (2) the influence of MVICES on the prediction of optimal muscle lengths of elbow flexors, and (3) the influence of neuromuscular activation on the prediction of optimal muscle length with MVIC.

\section{Materials and methods}

\subsection{Experimental method}

Eight subjects (five males and three females), with a mean age of 26.5 (20 to 34), and without previous history of neuromusculoskeletal or neuromuscular disorders volunteered to participate in this investigation. The anthropometric data were as follows: mean body height was $168.38 \mathrm{~cm}(161-177 \mathrm{~cm})$, mean body weight $60.06 \mathrm{~kg}$ (41-76 kg) and mean arm girth 27.43 $\mathrm{cm}(23-33.5 \mathrm{~cm})$. All of the subjects in this study had not involved in regular physical activity and did not consume any substances or food such as caffeine or alcohol before tests, which might affect the muscular performance.

An isokinetic dynamometer (Cybex Norm, Cybex, Division of Lumex, Inc., Ronkonkoma, NY, USA) was used to measure the flexion torques on the elbow joint. The test and fixation were after instructions in the operating manual given by the manufacture [12]. Subjects were secured with belt around pelvis. The axis of rotation of the elbow joint was aligned with the dynamometer's axis by adjusting the adapter length and the distance of seat from the dynamometer. The forearm was fully supinated and the shoulder was abducted at $15^{\circ}$. After being fixed with the positions as mentioned above, subjects were instructed to perform elbow flexion with the maximal voluntary isometric effort determined by the measured torque curve reaching its plateau. Eight positions of elbow flexion, from $15^{\circ}$ to $120^{\circ}$ at an interval of $15^{\circ}$ were investigated. The orders of the MVIC and MVICes as well as the test angles of elbow flexion were randomized assigned to avoid the effects of learning and carry-over. Following sessions of MVIC an electrical stimulator (CEFAR 
Medical AB, Lund, Sweden) was then superimposed to the maximal contraction of the elbow flexors. The pulse duration was $300 \mu \mathrm{sec}$ and the amplitude was set to induce maximal muscle contraction, sustained for 7 seconds and followed by a one-minute rest.

\subsection{Mathematical modeling}

An anatomical elbow model created by van Zuylen et al. [13] was adapted to calculate the moment arm and muscle length at different joint angles. The optimal length of elbow flexors was estimated using the optimization approach. A program in MATLAB (V $5.0,1997)$ was written for these calculations. The optimization problem formulated has the optimal length as its only design variable, ranging from the minimal length at maximal elbow flexion to the maximal length at full elbow extension. The cost function was set to minimize the sum of the differences of the joint torques between the experimentally measured and theoretically predicted joint torques as follows,

$$
\min . \sum_{i=1}^{n}\left(T_{i}-\tau_{i}\right)^{2}
$$

where $T_{i}$ and $\tau_{i}$ denotes the measured and predicted joint torques at the $i$ th joint position, respectively.

The model was customized to each individual subject by providing individualized physiologic cross section area (PCSA). The PCSA of individual subject $\left(\mathrm{PCSA}_{i}\right)$ was obtained by using the PCSA reported by An et al. [14] and was normalized by the cross sectional area of the arm (A) as follows.

$$
P C S A_{i}=P C S A_{a n} \times \frac{A i}{A m},
$$

where $A_{m}$ represents the mean of arm cross sectional area for all subjects; $A_{i}$ denotes the arm cross sectional area for the ith subject and calculated by

$$
A_{i}=\frac{C_{i}}{4 \pi}
$$

where $C_{i}$ denotes the circumference of the arm, which was determined by measuring the largest circumference of elbow flexors at 90 degrees of elbow flexion passively positioned.

There were three conditions considered, MVIC, MVIC $_{\mathrm{ES}}$, and MVIC with individualized levels of neuromuscular activation $\left(\mathrm{MVIC}_{\alpha}\right)$, to calculate the optimal lengths of elbow flexors. The individualized level of neuromuscular activation $(\alpha)$ shown in Eq. (1) was demonstrated as the ratio of joint torques generated in the condition of MVIC to those in the condition of
MVIC $_{\mathrm{ES}}$ assuming MVIC $\mathrm{ES}_{\mathrm{S}}$ elicited a full level of neuromuscular activation in which $\alpha$ was set to be one. In the present study, $\alpha$ in the condition of MVIC was assumed as one to interpret as a full level of neuromuscular activation as usual.

\subsection{Statistical analysis}

The statistic significance level was set at 0.05 . Paired $t$-test was used to identify the significance of differences in the joint torques and muscle length of elbow flexors predicted.

\section{Results}

The joint torques differed significantly between conditions with MVIC and MVIC $\mathrm{ES}_{\mathrm{S}}(p<0.05$, Table 1). The level of muscle activation derived from the ratio of measured joint torques was calculated, which ranged from 0.62 to 0.93 and averaged as 0.8 . The optimal muscle lengths of the elbow flexors (the biceps brachii, brachialis and brachioradialis) estimated were addressed in Table 2. The prediction of the optimal lengths of elbow flexors differed significantly between condition with MVIC and that with $\operatorname{MVIC}_{\mathrm{ES}}(p<$ $0.05)$, so as that existed between the condition of MVIC and $\mathrm{MVIC}_{\alpha}(p<0.05)$. Yet, there was no statistical difference between the conditions of $\mathrm{MVIC}_{\alpha}$ and that MVIC $_{\text {ES }}$. The estimation of the optimal lengths of brachialis and brachioradialis were significantly influenced by the condition with $\mathrm{MVIC}_{\mathrm{ES}}(p<0.05)$. The level of neuromuscular activation had the significant effect on the optimal length of brachialis in particular $(p<0.05)$.

\section{Discussion}

The joint torques recorded under condition of MVIC in the present study was smaller than those recorded under condition of $\mathrm{MVIC}_{\mathrm{ES}}$ similar to those reported by existing studies [8-10]. The difference in joint torques produced by $\mathrm{MVIC}_{\mathrm{ES}}$ from those produced by MVIC in most experiments demonstrated that neuromuscular tension was not entirely activated. The $\alpha$ derived from the joint torques varied individually, which indicated the individualized maximal capability of muscle contraction. The maximal torque generated in the condition of MVIC was approximately $80 \%$ of that generated 
Table 1

Joint torques generated $(\mathrm{N}-\mathrm{M})$ with and without electrical stimulation

\begin{tabular}{ccccc}
\hline Elbow flexion (degrees) & MVIC (N-M) & Confidence interval & MVIC $_{\mathrm{ES}}(\mathrm{N}-\mathrm{M})$ & Confidence interval \\
\hline 15 & 2.42 & $1.46-3.39$ & 2.96 & $1.84-4.08$ \\
30 & 2.48 & $1.61-3.34$ & 3.19 & $2.17-4.21$ \\
45 & 2.70 & $1.84-3.56$ & 3.39 & $2.42-4.37$ \\
60 & 2.84 & $2.13-3.55$ & 3.55 & $2.49-4.61$ \\
75 & 3.05 & $2.10-4.00$ & 3.74 & $2.82-4.67$ \\
90 & 3.12 & $2.39-3.84$ & 3.95 & $3.01-4.89$ \\
105 & 3.19 & $2.52-3.85$ & 3.97 & $2.83-5.10$ \\
120 & 2.72 & $2.29-3.15$ & 3.46 & $2.57-4.36$ \\
\hline
\end{tabular}

${ }^{*} p<0.001, p=6.33565391 \mathrm{E}-08$.

Table 2

Mean and (SD) of muscle length of elbow flexors $(\mathrm{cm})$ estimated under various conditions

\begin{tabular}{lccc}
\hline & Brachial biceps & Brachialis & Brachioradialis \\
\hline MVIC & $16.27(0.88)$ & $10.31(2.40)^{\mathrm{a}, \mathrm{b}}$ & $22.83(3.16)^{\mathrm{b}}$ \\
MVIC $_{\alpha}$ & $16.43(0.89)$ & $8.00(1.67)$ & $21.84(3.60)$ \\
MVIC $_{\mathrm{ES}}$ & $16.64(0.95)$ & $8.11(1.81)$ & $21.45(3.57)$ \\
\hline
\end{tabular}

${ }^{\mathrm{a}}$ A statistical difference in the muscle length at a level of significance of 0.05 in muscle length existed between the group with MVIC and those with MVIC $\alpha$.

${ }^{b}$ Statistical difference at a level of significance of 0.05 between the groups of $\mathrm{MVIC} \mathrm{ES}_{\mathrm{S}}$ and of MVIC.

through MVIC $\mathrm{ES}_{\mathrm{S}}$ and might correspond as submaximal muscle activation.

Consequently, the variations in the joint torques produced under different conditions might lead to statistical differences in the estimation of optimal muscle lengths. As a result of adjusting $\alpha$ individually in Eq. (1) to address the level of neuromuscular activation in the condition of MVIC, the optimal muscle lengths predicted were close to those derived from joint torques generated with $\mathrm{MVIC}_{\mathrm{ES}}$. Based on the results, it was concluded that it is improper to set unity as constant for maximal muscle activation during MVIC. Instead, taking 0.8 as the muscle activation level is considered in case of voluntary maximal contraction for the relevant research. Otherwise, obtaining MIVC through superimposed electrical stimulation might be considered.

Both of the brachialis and brachioradialis were affected by the condition of ES that demonstrated the role of primary elbow flexors and provided synergic contraction to assist biceps brachii and prevent undesired shoulder flexion motion $[15,16]$. The optimal length of brachialis was sensitive to the level of neuromuscular activation, which might imply its significance in the joint torques generation during elbow flexion without being considered by the adjacent joints such shoulder and wrist joints.

The present study was a preliminary study with relatively small sample size, which might limit the gen- eration of the results of this study and the confidence to approximate true population. There might be lower power to detect the variation of volitional effort of muscle activation. Furthermore, recording compound muscle activation potential (CMAP) caused by the changes of muscle length would be suggested to ensure maximal effort in addition to maximal torques recorded in this study.

\section{Conclusion}

Ideally, neuromuscular activation could be considered as unity with the condition of MVIC $\mathrm{ES}_{\mathrm{ES}}$. Or muscular activation should be proportionally modulated in the range of 0.62 to 0.93 during maximal voluntary muscle exertion alternatively. Both the brachialis and brachioradialis are significant in generating joint torques during elbow flexion. The present study suggests that the level of muscle contraction affects generation of joint torques that alters the muscle lengths and joint positions as well.

\section{References}

[1] R.D. Crowninshield and R.A. Brand, The prediction of forces in joint structures: Distribution of intersegamental resultants. Exercise and sport sciences reviews (ed. Miller, DI). The Franklin Institute Press, Philadelphia 9, 1981, pp. 159-181. 
[2] K.R. Kaufman, K.N. An and E.Y.S. Chao, Incorporation of muscle architecture into the muscle length-tension relationship, J Biomech 22 (1989), 943-948.

[3] W. Herzog, Muscle, in: Biomechnaics of the musculoskeletal system, B.M. Nigg and W. Herzog, eds, Chichester: John Wiley \& Sons, 1994, pp. 154-179.

[4] C.J. Zuurbier and P.A. Huijing, Changes in geometry of actively shortening unipennate rat gastrocnemius muscle, $J$ Morphol 218(2) (1993), 167-180.

[5] R.L. Lieber and J. Friden, Intraoperative measurement and biomechanical modeling of the flexor carpi ulnaris-to-extensor carpi radialis longus tendon transfer, J Biomech Eng 119(4) (Nov 1997), 386-391.

[6] A.A. Freehafer, P.H. Peckham and M.W. Keith, Determination of muscle-tendon unit properties during tendon transfer, J Hand Surg [Am] 4(4) (July 1979), 331-339.

[7] R.W. Ramsey and S.F. Street, Isometric length-tension diagram of isolated skeletal muscle fibers of frog, J Cell Comp Physiol 15 (1940), 11.

[8] Y.W. Chang, F.C. Su, H.W. Wu and K.N. An, Optimum length of muscle contraction, Clin Biomech 14 (1999), 537-542.

[9] J.J. Dowling, E. Konert, P. Ljucovic and D.M. Andrew, Are humans able to voluntarily elicit maximum muscle force? Neurosci Lett 179(1-2) (1994), 25-28.

[10] V. Strojnik, Muscle activation level during maximal voluntary effort, Eur J Appl Physiol Occup Physiol 72(1-2) (1995), 144-149.

[11] V. Strojnik, The effects of superimposed electrical stimulation of the quadriceps muscles on performance in different motor tasks, J Sports Med Phy Fitness 38(3) (1998), 194-200.

[12] Cybex Norm ${ }^{\mathrm{TM}}$ System User's Guide, Ronkonkoma, NY: Cybex, Division of Lumex, Inc., pp. 6-54.

[13] E.J. van Zuylen, A. van Velzen and J.J. Denier van der Gon, A biomechanical model for flexion torques of human arm muscles as a function of elbow angle, J Biomechanics 21 (1988), 183-190.

[14] K.N. An, F.C. Hui, B.F. Morrey, R.L. Linscheid and E.Y. Chao, Muscles across the elbow joint: a biomechanical analysis, $J$ Biomech 14(10) (1981), 659-669.

[15] L.K. Smith, E.L. Weiss and L.D. Lehmkuhl, Brunstrom's clinical kinesiology, 5th ed., Philadephia: F.A. Davis, 1996, p. 174.

[16] J.V. Basmajian and A. Latif, Integrated actions and functions of the chief flexors of the elbow: A detailed electromyographic analysis, J Bone Joint Surg [Am] 39 (1957), 1106. 